\title{
Optimierungsansätze für E-Mail-Kampagnen in der Kundenkommunikation
}

\author{
Robert Hauke, Klaus D. Wilde \\ Lehrstubl für Allgemeine Betriebswirtschaftslehre und Wirtschaftsinformatik, \\ Katholische Universität Eichstätt-Ingolstadt
}

\section{Einleitung}

In den letzten Jahren haben sich innerhalb der Kommunikation mit Kunden zahlreiche Änderungen vollzogen. Diese führten zu einem verstärkten Wettbewerb der Unternehmen um die Aufmerksamkeit ihrer Zielgruppe. Durch die weite Verbreitung des Internets haben sich neue Technologien (wie z. B. die E-Mail) als Kommunikationskanal in der Kundenkommunikation etabliert (Deutsche Post 2009, S. 65-67). Sie ermöglichen den Unternehmen eine kostengünstige und interaktive Kundenansprache.

Diese Änderung in der Kundenkommunikation führt zu einem steigenden Informationsangebot bei nahezu gleichbleibendem Medienkonsum der Zielgruppe. Durch den erhöhten Werbedruck werden weniger als $5 \%$ der Marketingkommunikation wahrgenommen (Bruhn 2007, S. 33). Neben der allgemeinen Informationsüberlastung beeinflussen noch weitere, kanalspezifische Aspekte die Wirkung der Kundenkommunikation. Im folgenden Beitrag wird die E-Mail als Kommunikationskanal der Kundenkommunikation näher untersucht.

Der Beitrag ist in fünf Kapitel gegliedert. Im nachfolgenden Abschnitt wird der aktuelle Forschungsstand im Themengebiet des Beitrags dargestellt und die Zielsetzung der Untersuchung abgeleitet. Kapitel 3 enthält die Beschreibung der Forschungsmethodik. Die Ergebnisse der empirischen Untersuchung werden in Kapitel 4 vorgestellt und diskutiert. Im abschließenden Kapitel 5 erfolgt eine kritische Würdigung der Untersuchungsergebnisse sowie eine Darstellung weiterführender Forschungsansätze.

\section{Problemstellung und Zielsetzung}

Obwohl die E-Mail als Kanal der Kundenkommunikation in der Praxis weit verbreitet ist, findet sie in der wissenschaftlichen Forschung bisher kaum Beachtung. Häufig handelt es sich um einzelne Untersuchungen innerhalb einer bestimmten 
Branche. Fuchs et al. (2008) und O'Connor (2008) untersuchen beispielhaft den Einsatz der E-Mail als Marketinginstrument in der Hotelindustrie. Rettie (2002) identifiziert im Rahmen einer Expertenbefragung ( $\mathrm{n}=4)$ die Bekanntheit des Absenders, die Betreffzeile sowie die inhaltliche und grafische Gestaltung als Faktoren, die das Reaktionsverhalten der Empfänger beeinflussen können. Die Allgemeingültigkeit und Vollständigkeit dieser Ergebnisse muss auf Grund der geringen Anzahl an Untersuchungsteilnehmern kritisch hinterfragt werden.

Angesichts dieses Forschungsstands strebt der vorliegende Beitrag eine umfassende Analyse zur Identifikation von Optimierungsansätzen in der Kundenkommunikation per E-Mail an. Dafür werden die Faktoren identifiziert, welche die Wirkung einer E-Mail-Kampagne beeinflussen können. Durch die Anzahl an Zustellungen, Öffnungen, Klicks und Abmeldungen kann die Wirkung einer E-MailKampagne gemessen werden. Weitere Transaktionen (z. B. Kauf) werden in der Untersuchung nicht berücksichtigt, da sie nicht unmittelbar durch die E-MailKampagne zu beeinflussen sind. Im Folgenden werden die einzelnen Kennzahlen auch als Response bezeichnet (siehe Abbildung 1).

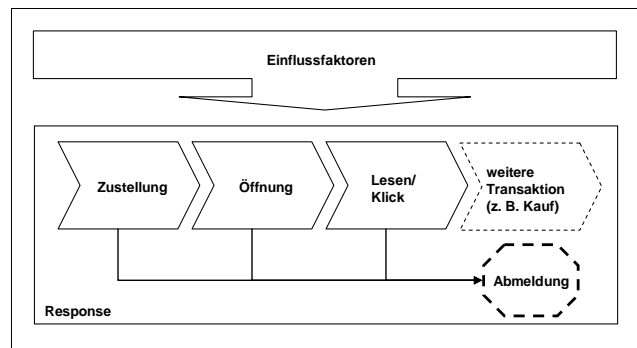

Abbildung 1: Responsekennzahlen von E-Mail-Kampagnen

\section{Vorgehen bei der Untersuchung}

Zur Identifizierung der Einflussfaktoren auf das Reaktionsverhalten der Empfänger von E-Mail-Kampagnen wurde eine Experten-Befragung durchgeführt. Da bisher kein gesichertes und vollständiges Wissen über die Einflussfaktoren besteht, wurde für die Untersuchung die Delphi-Methode verwendet. Diese mehrstufige Befragungsmethodik hat das Ziel, durch Rückkopplung der Ergebnisse eine Annäherung zwischen den Aussagen der Experten zu erreichen (Häder 2002, S. 24). Als Abbruchkriterium wurde die Konvergenz in den Interquartilsabständen gewählt; diese ist ein Indikator für den Grad des erreichten Konsens (siehe Abbildung 3).

Die Grundgesamtheit der Untersuchung besteht aus Personen, die über ein fundiertes Wissen im Bereich E-Mail-Marketing verfügen. Die Experten wurden anhand von Vorträgen, Veröffentlichungen und ihrer Position innerhalb des Unternehmens ausgewählt. In der ersten Runde nahmen 19 Experten aus unterschied- 
lichen Branchen an der Befragung teil. Insgesamt haben 17 Experten die Befragung abgeschlossen. Die heterogene Zusammensetzung der Experten aus unterschiedlichen Branchen ist wichtig, um die Fragestellung von unterschiedlichen Blickwinkeln zu betrachten und dadurch eine Allgemeingültigkeit der Ergebnisse zu erreichen (Schöllhammer 1970, S. 131).

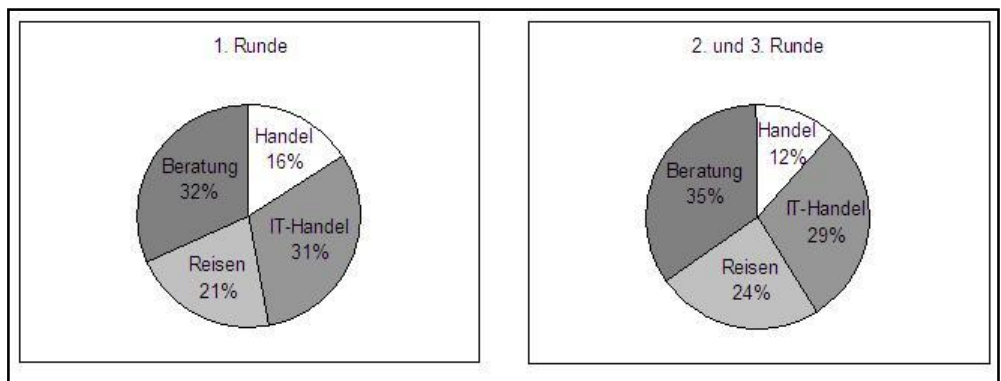

Abbildung 2: Teilnehmer und Branchenverteilung

In der ersten Befragungsrunde sollten die Experten im Rahmen von offenen Fragen die verschiedenen Faktoren identifizieren und ihren Einfluss auf das Reaktionsverhalten der Empfänger bewerten. In den darauffolgenden Runden wurden die gegebenen Antworten der Vorrunde klassifiziert und mit dem jeweiligen Median sowie den Interquartilsabständen in den Fragebogen aufgenommen. Die Experten erhielten dadurch die Möglichkeit, ihre Antworten zu validieren oder neue Einflussfaktoren hinzuzufügen. Da die Experten nach der zweiten Wiederholungsrunde bei ihren Antworten blieben und nur punktuell geringfügige Modifikationen bei einzelnen Antworten vornahmen, wurde die Delphi-Befragung nach der dritten Runde abgeschlossen.

\begin{tabular}{|c|c|c|c|c|c|c|c|c|c|}
\hline & \multicolumn{3}{|c|}{ 1. Runde } & \multicolumn{3}{c|}{ 2. Runde } & \multicolumn{3}{c|}{ 3. Runde } \\
\hline & $\begin{array}{c}\text { Quantile } \\
(25 \%)\end{array}$ & $\begin{array}{c}\text { Quantile } \\
(75 \%)\end{array}$ & Median & $\begin{array}{c}\text { Quantile } \\
(25 \%)\end{array}$ & $\begin{array}{c}\text { Quantile } \\
(75 \%)\end{array}$ & Median & $\begin{array}{c}\text { Quantile } \\
(25 \%)\end{array}$ & $\begin{array}{c}\text { Quantile } \\
(75 \%)\end{array}$ & Median \\
\hline Spamfilter & 4 & 5 & 5 & 4 & 5 & 4 & 4 & 4 & 4 \\
\hline
\end{tabular}

Abbildung 3: Konvergenz der Interquartilsabstände am Faktor Spam-Filter (1=sehr unwichtig $|\ldots| 5=$ sehr wichtig)

\section{Einflussfaktoren auf das Reaktionsverhalten der Empfänger}

Zur Strukturierung der Kommunikation sowie der Darstellung der Einflussfaktoren wird das Kommunikationsmodell von Lasswell (1964, S. 37) verwendet. Dabei übermittelt ein Absender (z. B. Unternehmen) eine Kommunikationsbotschaft über einen Kanal (z. B. E-Mail) einem oder mehreren Empfängern (z. B. Kunden). Die Kommunikationsbotschaft wird abhängig vom jeweiligen Übertragungskanal in Wort, Bild, Zeichen und/oder Musik kodiert. Dieser Kernprozess kann von 
situativen Störfaktoren beeinflusst werden (Bruhn 2007, S. 38-39). Die in der Delphi-Befragung identifizierten Einflussfaktoren können den einzelnen Elementen des Kommunikationsprozesses zugeordnet werden (siehe Abbildung 4).

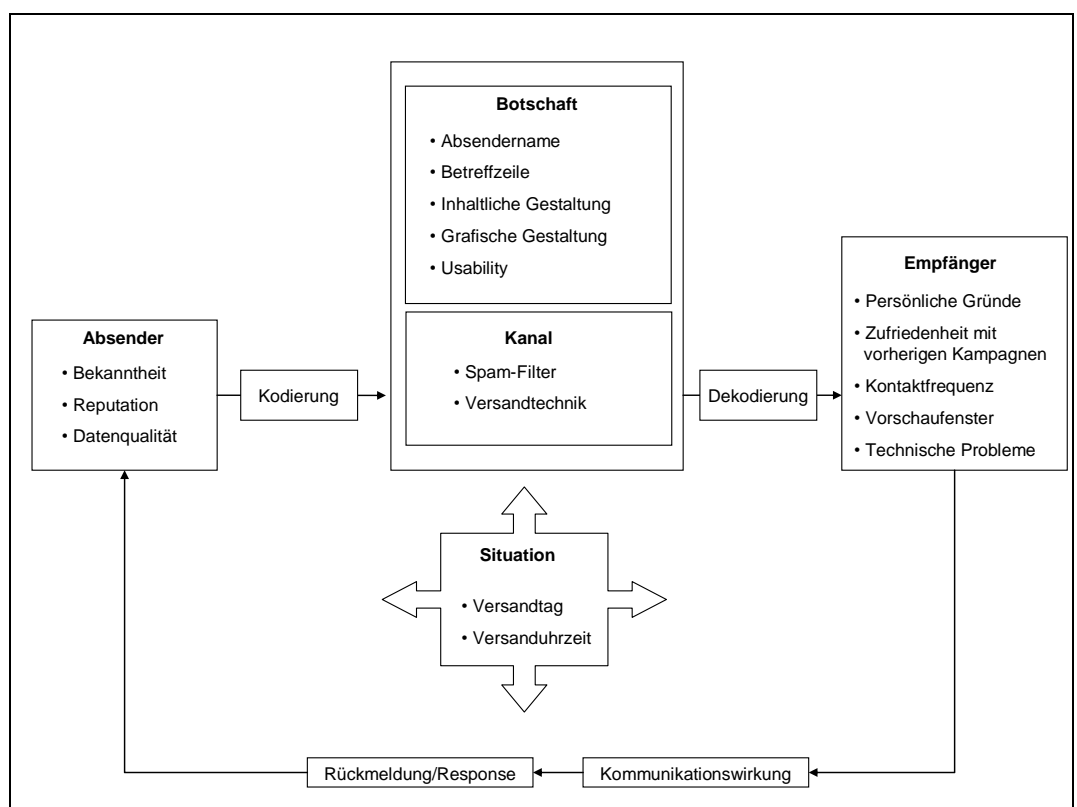

Abbildung 4: Ablauf der Kommunikation und Zuordnung der Einflussfaktoren (in Anlehnung an Bruhn 2007, S. 39)

Die Ergebnisse der Delphi-Befragung werden in den folgenden Abschnitten erläutert.

\subsection{Absenderbedingte Einflussfaktoren}

Die Experten schätzen die allgemeine Bekanntheit sowie die Reputation des Unternehmens als wesentliche Einflussfaktoren für das Responseverhalten der Empfänger ein. Insbesondere durch die wachsende Verbreitung von Spam-E-Mails, werden E-Mails unbekannter Absender zumeist direkt gelöscht (Aschoff 2005, S. 54). Die Bekanntheit sowie die Reputation des Unternehmens sind jedoch nicht allein durch E-Mail-Kampagnen beeinflussbar. Vielmehr ist es die Aufgabe einer kanalübergreifenden, integrierten Unternehmenskommunikation die Bekanntheit sowie die Reputation des Unternehmens zu stärken (Bruhn 2007, S. 89).

Ein weiterer wichtiger Einflussfaktor ist die Qualität der Kundendaten. Durch falsche Kundeninformationen (z. B. Anrede oder Titel) sinkt die Response der E-Mail-Kampagne. Zur Verbesserung der Datenqualität wurden durch die DelphiBefragung verschiedene proaktive und reaktive Maßnahmen identifiziert. 


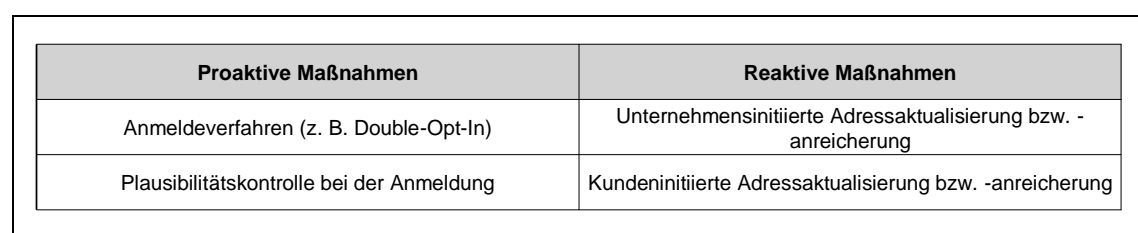

Abbildung 5: Maßnahmen zur Verbesserung der Datenqualität

Proaktive Maßnahmen setzen unmittelbar bei der Datenerfassung an. Bei der Anmeldung zum Newsletter-Service können die Eingabedaten des Kunden mit Hilfe von Plausibilitätskontrollen (z. B. existiert der Domain-Name im Internet) validiert werden.

Zudem kann bei der Anmeldung das Verfahren des Double-Opt-In angewandt werden. Hierbei erhält der Kunde im ersten Schritt eine Bestätigungs-E-Mail mit der Aufforderung auf Rückbestätigung, meist über einen in der E-Mail integrierten Link. Dabei hat er die Möglichkeit, seine Angaben zu überprüfen und zu ändern. Neben der Verbesserung der Datenqualität wird durch dieses Verfahren auch der Sorgfaltspflicht beim Versand von E-Mail-Kampagnen zu Werbezwecken entsprochen (BGH 2004).

Nach der Anmeldung des Kunden hat das Unternehmen verschiedene Möglichkeiten, reaktiv die Qualität der Kundendaten zu verbessern. Auf Seiten des Unternehmens können die Daten über interne Datenquellen oder über externe Kanäle (z. B. Listbroker) aktualisiert bzw. angereichert werden. Zudem können wiederholt nicht zustellbare E-Mail-Adressen (sog. Bounces) oder inaktive Empfänger (z. B. Kunden, die in den letzten zwölf Monaten auf keine E-MailKampagne reagiert haben), in der Datenbank bereinigt werden.

Durch kundeninitiierte Maßnahmen kann der Kunde selbstständig über das Internet (z. B. Unternehmenshomepage) oder über andere Kanäle (z. B. Call Center) seine Daten aktualisieren bzw. ergänzen.

\subsection{Botschaftsbedingte Einflussfaktoren}

Eine Kommunikationsbotschaft ist inhaltlich und grafisch so zu gestalten, dass sie die Aufmerksamkeit der Zielgruppe weckt und sie zu weiteren Handlungen (z. B. Kaufabschluss) bewegt. Im Rahmen der Delphi-Befragung gaben die Experten an, dass neben der inhaltlichen und grafischen Gestaltung, die Betreffzeile sowie der Name des Absenders das Reaktionsverhalten der E-Mail-Empfänger beeinflussen kann.

\section{Absendername}

Neben der in Kapitel 4.1 genannten Reputation und Bekanntheit des Absenders, hat die Gestaltung des Absendernamens einen wichtigen Einfluss auf das Respon- 
severhalten der Empfänger. Der Absender kann z. B. den Namen des Unternehmens eines persönlichen Ansprechpartners oder einen Markennamen enthalten. Beispielsweise verschickt Beiersdorf E-Mail-Kampagnen für die Marke Nivea direkt unter ihrem Markennamen.

Firmenname (PAYBACK/ PAYBACK.de)
\begin{tabular}{|l|l|l|l|}
\hline \multicolumn{1}{|c|}{ Allgemein } & \multicolumn{1}{|c|}{ Konkretes Thema } & \multicolumn{1}{|l|}{ Konkreter Personenname } \\
\hline $\begin{array}{l}\text { Beispiel: } \\
\text { PAYBACK Newsletter } \\
\text { PAYBACK News }\end{array}$ & $\begin{array}{l}\text { Beispiel: } \\
\text { PAYBACK Prämien } \\
\text { PAYBACK Online Shops } \\
\ldots\end{array}$ & $\begin{array}{l}\text { Beispiel: } \\
\text { Isabell Bach - PAYBACK.de }\end{array}$ \\
\hline
\end{tabular}

\section{Abbildung 6: Gestaltungsmöglichkeiten des Absenders}

Um den Absendernamen zu spezifizieren, können weitere Informationen, wie ein konkretes Thema oder der Name des Kundenbetreuers, ergänzt werden (siehe Abbildung 6). Bei der Gestaltung des Absendernamens ist zu beachten, dass nur eine begrenzte Anzahl an Zeichen je nach E-Mail-Provider bzw. Programm dargestellt wird.

\section{Betreffzeile}

Der Stellenwert der Betreffzeile wird in der Literatur oft hervorgehoben (Fuchs et al. 2008, S. 48; Rettie 2002, S. 7). Auch die befragten Experten erachten den Einfluss der Betreffzeile auf das Reaktionsverhalten der Empfänger als sehr wichtig. Um die Wirkung der Betreffzeile auf das Responseverhalten der Empfänger zu untersuchen, müssen die verschiedenen Gestaltungsmöglichkeiten einer Betreffzeile klassifiziert werden. Im Rahmen einer qualitativen Inhaltsanalyse wurden neun Klassifizierungsmerkmalen hergeleitet, die sich nach Inhalt, Stil und Format unterscheiden (Hofinger 2008, S. 11-21). Im Folgenden werden die einzelnen Gestaltungsmerkmale kurz erläutert.

\begin{tabular}{|c|c|c|}
\hline \multicolumn{3}{|c|}{} \\
\hline Inhalt & Stil & Format \\
\hline Limitierung & $\begin{array}{c}\text { Formulierung } \\
\text { (z. B. informativ, emotional) }\end{array}$ & Länge \\
\hline $\begin{array}{c}\text { Anreiz } \\
\text { Stilmittel } \\
\text { (z. B. Rabattaktion, Gewinnspiel) }\end{array}$ & (z. B. Metapher, Alliteration) & $\begin{array}{c}\text { Optische Hervorhebung } \\
\text { (z. B. Sonderzeichen) }\end{array}$ \\
\hline Persönliche Ansprache & Konkretisierungsgrad & Aufzählungsliste \\
\hline
\end{tabular}

Abbildung 7: Gestaltungsmöglichkeiten der Betreffzeile 
Anreize, Limitierungen sowie die persönliche Ansprache gelten als gängige Stimuli von Mailings, um die Empfänger zu aktivieren und die Response des Mailings zu steigern (Bruhn 2007, S. 365-385).

Die Limitierung von Inhalten wird eingesetzt, um den Empfänger zu einem schnellen Handeln aufzufordern. Dabei kann die Menge (z. B. „Nur 99 Angebote verfügbar") oder der Aktionszeitraum des Angebots (z. B. „Sparen Sie bis zu 50 \% an Ostern") limitiert werden. Anreize können in Form von Rabattaktionen oder Gewinnspielen gegeben werden. Durch die persönliche Ansprache soll dem Empfänger Exklusivität der Kommunikationsbotschaft vermittelt werden. Dabei kann der Empfänger entweder direkt mit seinem Namen oder indirekt mit einem Personalpronomen angesprochen werden.

Neben den inhaltlichen Kriterien können stilistische Kriterien zur Gestaltung der Betreffzeile verwendet werden. Diese betreffen die Formulierung (z. B. emotional) der Betreffzeile. Durch den Einsatz von Stilmitteln (z. B. Metapher oder Humor), respektive rhetorischen Figuren, können Texte interessanter, lebendiger und anschaulicher gestaltet werden (Eisend und Kuß 2009, S. 631-639). Mit Hilfe des Konkretisierungsgrads der Betreffzeile kann entweder die Neugier des Empfängers geweckt werden oder der Empfänger umfassend über sämtliche Inhalte der Werbebotschaft informiert werden.

Ein weiteres Unterscheidungskriterium ist das Format. Optische Hervorhebungen zur Gestaltung der Betreffzeile stehen nur in begrenztem Umfang zur Verfügung. Während beispielsweise der Einsatz von Farbe oder Hintergrunddesign nicht möglich ist, können z. B. Anführungszeichen oder Textsymbole eingesetzt werden. Durch die Verwendung einer Aufzählungsleiste - i. d. R. werden einzelne Schlagwörter aneinander gereiht - können mehrere Themen in der Betreffzeile aufgegriffen werden. Der Empfänger erhält somit einen umfassenden Überblick über die verschiedenen Inhalte der E-Mail-Kampagne. Wie bei der Gestaltung des Absenders ist auch bei der Betreffzeile zu beachten, dass die verschiedenen E-Mail-Provider (z. B. Hotmail) und E-Mail-Programme (z. B. Outlook) die Anzahl der angezeigten Zeichen unterschiedlich begrenzen.

\section{Inhaltliche Gestaltung}

Die Inhalte der E-Mail sollten an den individuellen Bedürfnissen des Empfängers ausgerichtet sein. Empfindet der Empfänger die Kommunikationsbotschaft als nicht relevant, sinkt sein Interesse, auf die E-Mail-Kampagne zu reagieren.

Im Allgemeinen ist zwischen informativen und emotionalen Inhalten zu unterscheiden. Informative Inhalte stellen objektive Tatbestände oder Produkteigenschaften bzw. -vorteile in den Vordergrund. Dies ist vor allem in Märkten mit hochkomplexen Leistungen oder reiner Preisorientierung anzutreffen. In Märkten mit hohem Kommunikationswettbewerb wird vermehrt auf emotionale Inhalte zurückgegriffen, da sie größeren Einfluss auf die Aufmerksamkeit der Empfänger ausüben (Meffert et al. 2008, S. 711-717). 


\section{Grafische Gestaltung}

Die grafische Gestaltung bezieht sich auf die Struktur sowie das Design der E-Mail-Kampagne. Beide Aspekte wurden in der Delphi-Befragung als wichtig beurteilt, um den Empfänger zum aufmerksamen Lesen der Kampagne zu motivieren. Die Struktur legt fest, wie die einzelnen Inhalte bzw. Elemente einer EMail-Kampagne angeordnet sind. Das Design hingegen umfasst die Anzahl und Art der Bilder, die Gestaltung der Links sowie die Darstellung der Schrift (Art, Größe). Dabei sind auch Aspekte der Usability zu berücksichtigen.

Der Verwendung von grafischen Elementen (z. B. Bilder) kommt bei der Gestaltung des Designs eine zentrale Rolle zu. Bilder werden schneller als Texte wahrgenommen und bewirken zudem eine höhere Erinnerungskraft beim Empfänger (Meffert et al. 2008, S. 709-711). Allerdings sollten Bilder eine E-Mail nicht überladen. $\mathrm{Zu}$ viele visuelle Reize steigern die Komplexität und schränken die Übersichtlichkeit der Kampagne ein (Geissler et al. 2006, S. 74-75).

\subsection{Kanalbedingte Einflussfaktoren}

Mit Hilfe der E-Mail als Kommunikationskanal wird die Botschaft vom Unternehmen an die Empfänger übermittelt. Hierbei beurteilen die Experten die Versandtechnik sowie Spam-Filter als sehr wichtige Einflussfaktoren.

Als Versandtechnik setzen Unternehmen i. d. R. eine E-Mail-Marketing Software ein. Diese ist entweder im Unternehmen selbst installiert, oder wird bei einem externen Versanddienstleister gehostet. Nach Auskunft der Experten handelt es sich häufig um eine „Insellösungen“, die über Schnittstellen an die unternehmensinterne IT-Infrastruktur (z. B. CRM-System) angebunden ist. Um die Zustellbarkeit der Kampagne zu sichern, sollte die E-Mail vor Versand auf spamgefährdete Merkmale überprüft werden. Im Wesentlichen existieren zwei verschiedene Arten von Spam-Filtern:

IP-Filter überprüfen die IP-Adresse des Absenders. Ist die Adresse in einer Black-List eingetragen, wird die E-Mail nicht zugestellt.

Content-Filter überprüfen die E-Mail-Kampagne auf verdächtige Merkmale. Neben den Kampagneninhalten wird u. a. die Programmierung kontrolliert. Entspricht die Programmierung nicht den W3C-Richtlinien kann die E-Mail ebenfalls als Spam klassifiziert werden.

\subsection{Empfängerbedingte Einflussfaktoren}

Empfängerbedingte Einflussfaktoren betreffen die Fähigkeit des Empfängers, die Kommunikationsbotschaft der E-Mail-Kampagne zu verarbeiten und zu speichern. Voraussetzung hierfür ist die Dekodierung der Kommunikationsbotschaft $-\mathrm{d}$. h. die E-Mail muss vom Empfänger entschlüsselt und verstanden werden (Bruhn 2007, S. 39-41). Jeder Mensch hat auf Grund seiner psychischen und physischen 
Merkmale (Alter, Bildungsniveau etc.) ein individuelles Wahrnehmungs- und Verarbeitungsverhalten (Meffert et al. 2008, S. 704). Daher sollte die E-MailKampagne auf die Fähigkeiten der einzelnen Empfänger angepasst sein. So können sich z. B. die Inhalte und die grafische Gestaltung abhängig vom Bildungsniveau und Alter der Empfänger unterscheiden (Meffert et al. 2008, S. 710).

Des Weiteren können (bisherige) Erfahrungen des Kampagnenempfängers mit dem werbenden Unternehmen sein Reaktionsverhalten beeinflussen. Unzufriedenheit mit der bisherigen Kontaktfrequenz oder den Kampagneninhalten rufen negative Einstellungen gegenüber dem Absender hervor (Bruhn 2007, S. 47).

Die inhaltliche und grafische Personalisierung der E-Mail-Kampagne bewerten die Experten der Delphi-Befragung als sehr wichtig. Die Faktoren „Unzufriedenheit mit ehemaligen Inhalten“ sowie „Ansprachehäufigkeit" sind hingegen von geringerer Bedeutung.

\subsection{Situationsbedingte Einflussfaktoren}

Situationsbedingte Faktoren beziehen sich auf Einflüsse, die das Unternehmen nicht selbst oder nur teilweise steuern kann. Dazu zählen beispielsweise der Zeitdruck des Empfängers, die Ablenkung durch andere Werbemaßnahmen oder die Jahreszeit (z. B. Weihnachten).

Diese Faktoren können teilweise durch den Versandzeitpunkt (Tag, Uhrzeit) beeinflusst werden. Der Versandzeitpunkt muss jedoch nicht gleichzeitig mit dem Empfangszeitpunkt der E-Mail-Kampagne übereinstimmen, da die Empfänger z. T. den Posteingang nicht regelmäßig abrufen. Durch die schwierige Steuerbarkeit der situationsbedingten Faktoren ist die Wirkung des Versandzeitpunkts in der Literatur umstritten (Wegener 2006, S. 413). Im Rahmen der Delphi-Befragung wurde die Versanduhrzeit als wichtig, hingegen der Tag als nur mittelmäßig wichtig beurteilt.

Zur Bestimmung des Versandzeitpunkts ist das Internetnutzungsverhalten der Empfänger zu untersuchen. So kann z. B. die Anzahl der Aufrufe einer Unternehmenshomepage ein Indiz liefern, zu welchem Zeitpunkt ein besonderes Interesse an dem jeweiligen Unternehmen besteht.

\section{$5 \quad$ Fazit und Ausblick}

Im Rahmen der Delphi-Befragung wurden verschiedene Faktoren identifiziert und bewertet, die aus Sicht der Experten die Wirkung einer E-Mail-Kampagne beeinflussen können. Die Zahlen stellen den Median der Befragungsergebnisse dar (siehe Abbildung 8). 


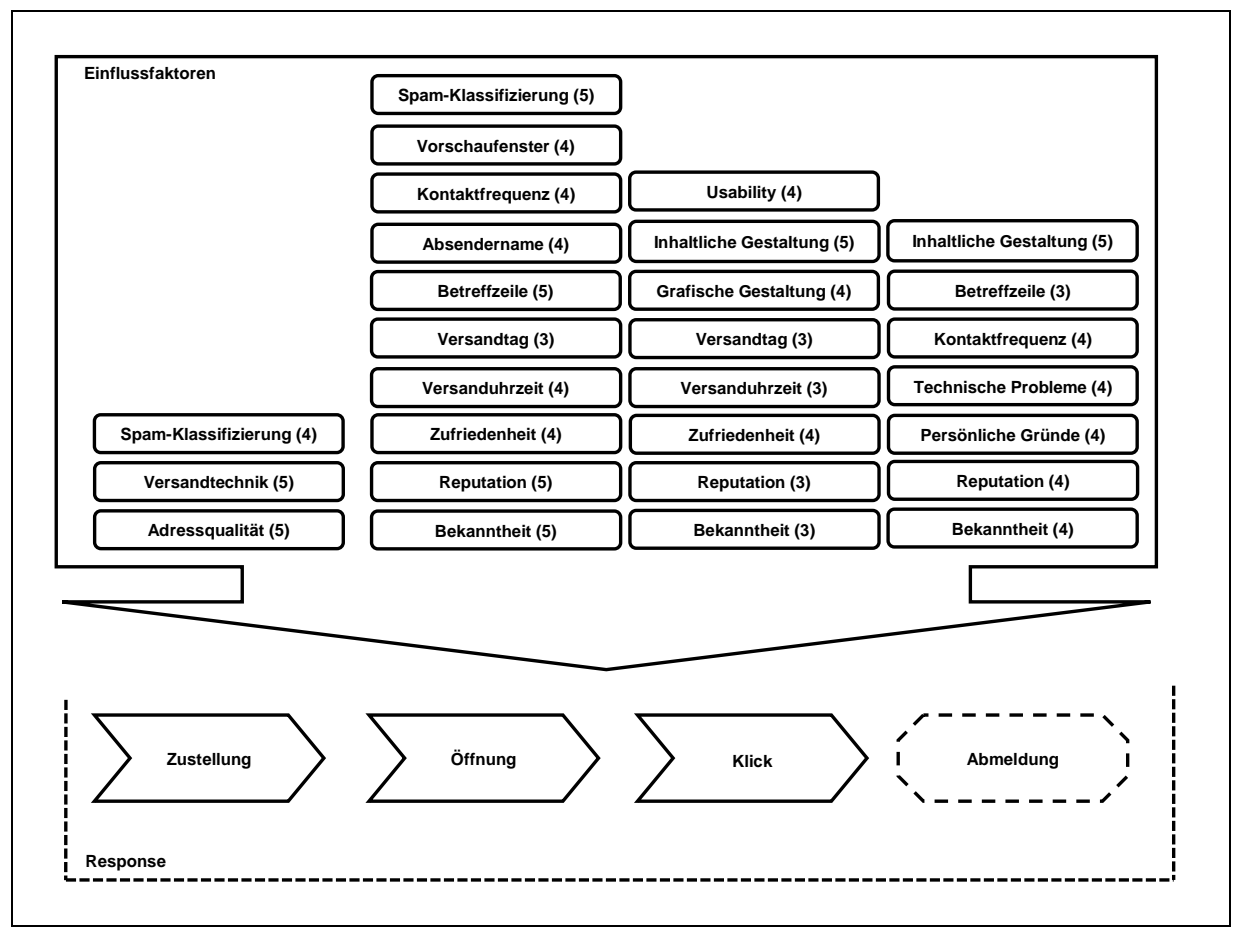

Abbildung 8: Responsemodell (1=sehr unwichtig $|\ldots| 5=$ sehr wichtig)

Im Rahmen zukünftiger Untersuchungen können die identifizierten Einflussfaktoren durch eine branchen- bzw. unternehmensspezifische Betrachtung weiter verfeinert werden. Insbesondere die Überprüfung der Einflussfaktoren durch experimentelle Feldbeobachtungen (z. B. Testkampagnen), kann die tatsächliche Relevanz offenlegen. Somit können Unternehmen die zentralen Einflussfaktoren identifizieren und die Wirkung zukünftiger E-Mail-Kampagnen steigern.

\section{Literatur}

Aschoff M (2005) Professionelles Direkt- und Dialogmarketing per E-Mail. Hanser, München.

BGH (2004) E-Mail-Werbung Leitsatzentscheidung (I ZR 81/01). http://www.bundesgerichtshof.de. Abruf am 2009-09-28.

Böhler H (1997) Marktsegmentierung als Basis eines Direct-Marketing-Konzepts. In: Dallmer H (Hrsg) Handbuch Direct Marketing. Gabler, Wiesbaden.

Bruhn M (2007) Kommunikationspolitik. Vahlen, München. 
Deutsche Post (2009) Dialog Marketing Monitor. http://www.deutschepost.de. Abruf am 2009-09-28.

Eisend M, Kuß A (2009) Humor in der Kommunikation.

In: Bruhn M; Esch FR; Langner T (Hrsg) Handbuch Kommunikation. Gabler, Wiesbaden.

Fuchs M; Tuta M; Höpken W (2008) Adoption of E-mail Marketing in the Hotel Sector. In: O'Connor P; Höpken W; Gretzel U (Hrsg) Information and Communication Technologies in Tourism 2008. Springer, Wien.

Geissler GL; Zinkhan GM; Watson RT (2006) The Influence of Homepage Complexity on Consumer Attention, Attitudes and Purchase Intent. Journal of Advertising 35 (2): 69-80.

Häder M (2002) Delphi-Befragungen - Ein Arbeitsbuch.

Westdeutscher Verlag, Wiesbaden.

Hofinger S (2008) Formen der Betreffzeilengestaltung im E-Mail-Marketing. Seminararbeit am Lehrstuhl für ABWL und Wirtschaftsinformatik, Katholische Universität Eichstätt-Ingolstadt.

Lasswell HD (1964) The Structure and Function of Communication in Society. In: Bryson L (Hrsg) Communication of Ideas, New York, S. 37-51.

Meffert H; Burmann C; Kirchgeorg M (2008) Marketing - Grundlagen marktorientierter Unternehmensführung. Gabler, Wiesbaden.

O'Connor P (2008) E-Mail Marketing by International Hotel Chains: An IndustryPractices Update. In: Cornell Hospitality Quarterly 49 (1): 42-52.

Rettie R (2002) Email Marketing: Success Factors. http://ausweb.scu.edu.au/aw02/papers/refereed/rettie/paper.html. Abruf am 2009-09-28.

Schöllhammer H (1970) Die Delphi-Methode als betriebliches Prognose- und Planungsverfahren. Schmalenbachs Zeitschrift für betriebswirtschaftliche Forschung 22 (2): 128-137.

Wegener M (2006) Online-Marketing-Controlling. In: Zerres C; Zerres MP (Hrsg.): Handbuch Marketing-Controlling. Springer, Berlin. 